Research Article

\title{
Quantitative Analysis of Flavonoids in Glycyrrhiza uralensis Fisch by ${ }^{1} \mathrm{H}$-qNMR
}

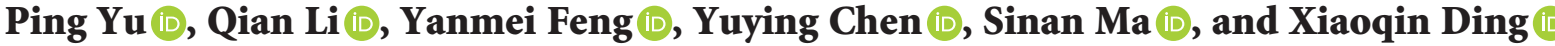

Gansu Provincial Key Laboratory of Aridland Crop Science, College of Agronomy, Gansu Agricultural University, Lanzhou 730070, China

Correspondence should be addressed to Qian Li; liqian1984@gsau.edu.cn

Received 12 October 2020; Revised 19 December 2020; Accepted 8 January 2021; Published 18 January 2021

Academic Editor: Ilari Filponnen

Copyright $(92021$ Ping Yu et al. This is an open access article distributed under the Creative Commons Attribution License, which permits unrestricted use, distribution, and reproduction in any medium, provided the original work is properly cited.

Objective. To establish a method for simultaneous determination of liquiritin, liquiritigenin, and isoliquiritinin glycyrrhizin using hydrogen nuclear magnetic resonance quantitative technology ( ${ }^{1} \mathrm{H}$-qNMR). Methodology. Deuterated dimethyl sulfoxide was used as the solvent, and dichloromethane was used as the internal standard. The probe temperature was $298.0 \mathrm{~K}$, the pulse sequence was $\mathrm{Zg} 30$, the number of scans was 16 , and relaxation delay $(D 1)$ was $10 \mathrm{~s}$. Quantitative characteristic signal peaks were $\delta$ $4.891 \sim 4.878 \mathrm{ppm}, \delta 8.187 \sim 8.172 \mathrm{ppm}$, and $\delta 6.790 \sim 6.776 \mathrm{ppm}$ for liquiritin, isoliquiritin, and liquiritigenin, respectively. Results. The experimental result showed that the content of flavonoids in Licorice, from Chifeng, Inner Mongolia, was the highest. Conclusion. In this study, a new method for determination of three flavonoids in Licorice using ${ }^{1} \mathrm{H}$-qNMR was established. This experimental method has the advantages of accuracy, efficiency, and economy. It lays a foundation for the study on the determination of flavonoids content in licorice by proton nuclear magnetic resonance spectroscopy.

\section{Introduction}

Glycyrrhiza uralensis Fisch. is a popular Chinese herbal medicine derived from the dried roots and rhizomes of Glycyrrhiza uralensis Fisch. species (Leguminosae family) [1]. It is one of the oldest and most popular herbal medicines in the world and was recorded in many Asian and European pharmacopoeias including China. In China, Glycyrrhiza uralensis Fisch. is called Gan-Cao, which means "sweet weed". As a tonifying herbal medicine, Gan-Cao is extensively used in the traditional Chinese medicine (TCM) and appears as a component herb in about $60 \%$ of all TCM prescriptions [2]. In addition to medicinal usage, Gan-Cao is also used as a cake additive in food; its sweetness is onehundred times that of sucrose. It also has applications in cosmetics, tobacco, and animal husbandry, etc. Gan-Cao shows a variety of pharmacological activities, including antiulceric, antiinflammatory, antispasmodic, antioxidative, antiallergic, antiviral, antidiabetic, anticancer, antidepressive, hepatoprotective, expectorant, and memory enhancing activities [3-5]. It is mainly used in clinical treatment of inflammation, cardiovascular, and cerebrovascular diseases, oxidative aging, tumors, etc [6-9]. The main active ingredients of Glycyrrhiza uralensis Fisch. include Glycyrrhiza uralensis Fisch. saponin and Glycyrrhiza uralensis Fisch. Flavonoids [10] and the liquiritin, liquiritigenin, and isoliquiritin are the main components of flavonoids. Although Glycyrrhiza uralensis Fisch. is considered as Generally Recognized as Safe (GRAS) for use in food by FDA (21 CFR 184.1408), large amounts may result in severe hypertension, hypokalemia, and other signs of mineralocorticoid excess $[11,12]$. Therefore, quality control is critical to ensure the efficacy and safety of Glycyrrhiza uralensis Fisch.

Therefore, the content of liquiritin, liquiritigenin, and isoliquiritin in Glycyrrhiza uralensis Fisch. was determined in this study. Analytical methods of Glycyrrhiza uralensis Fisch. flavonoids include high-performance liquid chromatography (HPLC), thin-layer chromatography (TLC), and gas chromatography (GC). Some people also used gravimetric methods to measure the content of Glycyrrhiza uralensis Fisch. Flavonoids [13]. HPLC and GC are the most commonly used methods for quality control of TCM $[14,15]$. However, their detectors are dependent on differing 
physical properties of the analytes. Thus, quantitative analysis of Glycyrrhiza uralensis Fisch. using these traditional methods requires two or more instruments. Considering the high requirement for efficacy and safety of Glycyrrhiza uralensis Fisch., an easier and faster method is needed for routine quality control [16].

Nuclear magnetic resonance (NMR) spectroscopy is commonly used for structure elucidation, but the potential for its quantitative ability is increasing nowadays. The ${ }^{1} \mathrm{H}-\mathrm{qNMR}$ method could simultaneously detect multiple components in very short time (1-5 mins) using a very cheap internal standard reference, which has found applications in quantitative analysis of natural products and other areas [12, 17-34]. In our previous study, we have developed a quantitative NMR (qNMR) method for Angelica dahurica and Angelicae Pubescentis Radix analysis [35, 36]. Major peaks in ${ }^{1} \mathrm{H}-\mathrm{NMR}$ and MS spectra contributing to the discrimination among species were assigned as those of glycyrrhizin, 4hydroxyphenyl acetic acid, and glycosidic conjugates of liquiritigenin/isoliquiritigenin.

In previous studies, Farag et al. reported the metabolite profiling and fingerprinting of medicinal licorice roots [37], and the ${ }^{1} \mathrm{H}-\mathrm{NMR}$ method was used to qualitatively identify the unknown components of the established fingerprints. Glycyrrhiza uralensis Fisch was investigated though centrifugal partition chromatography by Simmler et al. [38], and qNMR was used to determine the purity and residual complexity of the isolated compounds through orthogonal analysis. As known to us, there have been no reports about the simultaneous determination of liquiritin, liquiritigenin, and isoliquiritin by qNMR method, which are the active constituents of Glycyrrhiza uralensis Fisch. Therefore, the purpose of this study was firstly to simultaneously quantify the three main components liquiritin, liquiritigenin, and isoliquiritin. in Glycyrrhiza uralensis Fisch. by ${ }^{1} \mathrm{H}$-qNMR. The structural formula of the three components is shown in Figure 1.

\section{Materials and Methods}

2.1. Materials and Reagents. The reference standard of liquiritin ( $\geq 98 \%$ ), liquiritigenin ( $\geq 98 \%)$, and isoliquiritin $(\geq 98 \%$ ) was purchased from Chengdu coming DE biological technology co., LTD (Sichuan, China). The internal standard dichloromethane $(\geq 99 \%)$ was purchased from Sinopharm Group Chemical Reagent Co., Ltd. (Shanghai, China). DMSO- $_{6}(>99.8 \%)$ was purchased from Cambridge Isotope Laboratories, Inc. Other chemicals used in this work were of analytical grade.

The plant materials of Glycyrrhiza uralensis Fisch. were collected from Chifeng (Inner Mongolia, China), Hangqi (Inner Mongolia, China), Baotou (Inner Mongolia, China), Yanchi (Ningxia, China), Longxi (Gansu, China), and Taklamakan Desert (Xinjiang, China) and were identified as a piece of dried root and rhizome of the legume Glycyrrhiza uralensis by Professor Chen Yuan from the Department of Chinese Herbal Medicine of Gansu Agricultural University. The plant material samples were smashed with a pulverizer and passed through a 40-mesh sieve. The dried root sample used to determine repeatability, stability, and recovery was collected from Chifeng (Inner Mongolia, China).

2.2. Instrumentation. The nuclear magnetic resonance spectrometer (Bruker Advance III 600); AL-104 electronic analytical balance (Cixi Tiandong Weighing Apparatus Factory); KQ-500B ultrasonic cleaning instrument (Shenzhen Dekang Technology Co., Ltd.); rotary evaporator R-101N (Zhengzhou Great Wall Science and Industry Co., Ltd.); and constant temperature water bath (Jiangsu Zhengji Co., Ltd.) were used.

\subsection{NMR Analytical Method Development}

(1) Preparation of inner standard solution: $100 \mu \mathrm{L}$ dichloromethane was dissolved in $5 \mathrm{~mL}$ DMSO- $\mathrm{d}_{6}$, and an internal standard solution of $26.5 \mathrm{mg} / \mathrm{mL}$ was prepared.

(2) Preparation of samples for ${ }^{1} \mathrm{H}-\mathrm{qNMR}$ analysis: we have adopted the Chinese Pharmacopoeia Commission extraction method [38] for sample preparation. Decoction pieces of Glycyrrhiza uralensis Fisch. were crushed in a pulverizer and passed through a 40-mesh sieve. $10 \mathrm{~g}$ of Glycyrrhiza uralensis Fisch. powder was precisely weighed and put it in ethyl acetate and back flow for $1 \mathrm{~h}$ (material-liquid ratio $1: 20 \mathrm{~g} / \mathrm{mL}$ ). Then, the filter residue was taken and put it in methanol back flow for $1 \mathrm{~h}$ (materialliquid ratio $1: 15 \mathrm{~g} / \mathrm{mL}$ ). The filtrate was made into an extract and dissolved in water. The solution was washed three times with n-butyl alcohol, and the filtrates were combined to make extract.

(3) Preparation of standard solution: we followed the methods of [22], $5 \mathrm{mg}$ of isoliquiritin, liquiritigenin, and liquiritin were accurately weighed and put in DMSO- $_{6}(480 \mu \mathrm{L})$ and $20 \mu \mathrm{L}$ dichloromethane solution (internal standard reference) were added, respectively. The standard solutions of isoliquiritin, liquiritigenin, and liquiritin were obtained, respectively. $30 \mathrm{mg}$ of extract was accurately weighed and dissolved in $480 \mu \mathrm{L}$ DMSO- $\mathrm{d}_{6}$, and $20 \mu \mathrm{L}$ methylene chloride solution was added. The NMR spectra of extract and isoliquiritin, liquiritigenin, and liquiritin are shown in Figure 2.

2.4. ${ }^{1} \mathrm{H}$-NMR Spectroscopy. In this work, a $600 \mathrm{MHz}$ NMR spectrometer was used to obtain the ${ }^{1} \mathrm{H}$-NMR spectra data, and the data were analyzed with MestReNova software. Using the experimental model of [26] to optimize the acquisition conditions and follow the contents: the spectral width: $11904 \mathrm{~Hz}$, acquisition time: $3.78 \mathrm{~s}, D 1: 10 \mathrm{~s}$, the pulse sequence was $\mathrm{Zg} 30$, the number of scans was 16 , and the probe temperature was $298.0 \mathrm{~K}$.

2.5. Methodology Validation. In this work, the following parameters were used to validate the developed method: linearity, limit of detection, limit of quantitation, precision, repeatability, stability, and recovery. 


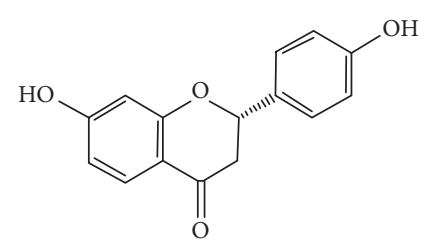

(a)

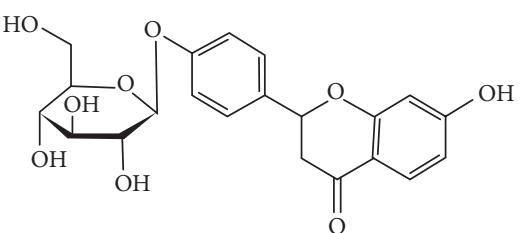

(b)<smiles>O=C(/C=C/c1ccc(O[C@@H]2OC(CO)[C@H](O)[C@H](O)[C@H]2O)cc1)c1ccc(O)cc1O</smiles>

(c)

Figure 1: Structures of the reference standard (flavonoids): (a) liquiritigenin, (b) liquiritin, and (c) isoliquiritin.

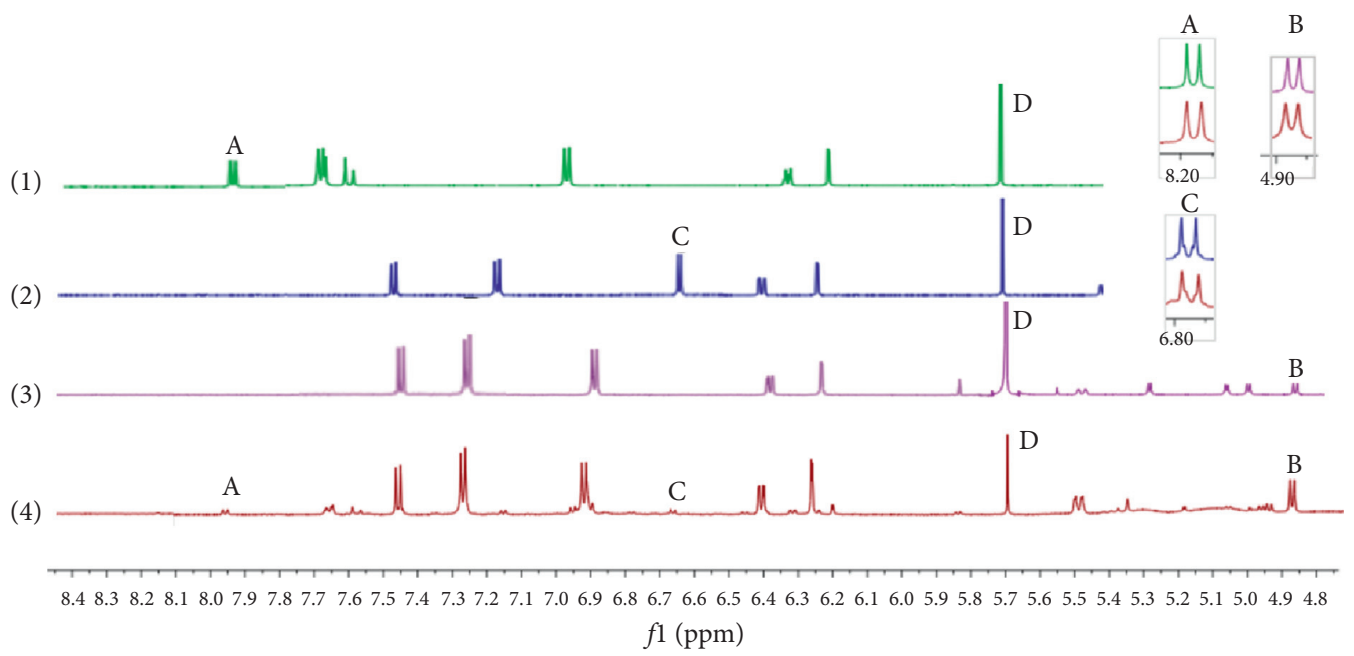

FiguRE 2: ${ }^{1} \mathrm{H}$-NMR spectra of isoliquiritin (1), liquiritigenin (2), liquiritin (3), and Glycyrrhiza uralensis Fisch. Extract (4), obtained at $600 \mathrm{MHz}$ in DMSO- $\mathrm{d}_{6}$ solvent. The quantitative signals of isoliquiritin (A), liquiritin (B), liquiritigenin (C), and dichloromethane (D) were at $\delta 8.187-8.172 \mathrm{ppm}, \delta 4.891-4.878 \mathrm{ppm}, \delta 6.83-6.85 \mathrm{ppm}$, and $\delta 5.75 \mathrm{ppm}$, respectively.

The linearity of liquiritin, isoliquiritin, and liquiritigenin is shown in Table 1, which indicated that the constructed analytical curves presented a satisfactory linearity.

The limit of detection and quantitation can be determined by the methods of [22]. $\mathrm{LOD}=3.3 \sigma / \mathrm{s}$ and $\mathrm{LOQ}=10 \sigma / \mathrm{s}$. The detection and quantitation limits of liquiritin were $0.023 \mathrm{mg} /$ $\mathrm{mL}$ and $0.070 \mathrm{mg} / \mathrm{mL}$, the detection and quantitation limits of liquiritigenin were $0.022 \mathrm{mg} / \mathrm{mL}$ and $0.068 \mathrm{mg} / \mathrm{mL}$, and the detection and quantitation limits of isoliquiritin were $0.003 \mathrm{mg} / \mathrm{mL}$ and $0.010 \mathrm{mg} / \mathrm{mL}$, respectively.

The same reference standard was measured six times to get precision results; six sample solutions were used to get repeatability results, and the same sample was measured within $12 \mathrm{~h}$ to get the stability results. The standard deviations of precision for liquiritin, isoliquiritin, and liquiritigenin were $1.18 \%, 1.13 \%$, and $1.24 \%$, respectively. The standard deviations of repeatability for liquiritin, isoliquiritin, and liquiritigenin were $1.68 \%, 2.29 \%$, and $4.43 \%$, respectively. The standard deviations of stability for liquiritin, isoliquiritin, and liquiritigenin were $1.08 \%, 2.25 \%$, and $1.24 \%$, respectively.

Three equal amounts of licorice extracts were accurately weighed, and the contents of liquiritin, liquiritigenin, and isoliquiritinin were calculated. Then, the double amount of standard solution was added to the licorice extracts solution, and the recovery results are shown in Table 2.
TABLE 1: Standard curves of three flavonoids in Glycyrrhiza uralensis Fisch.

\begin{tabular}{lccc}
\hline Compound & Regression equation & $R^{2}$ & Linearity $(\mathrm{mg} / \mathrm{ml})$ \\
\hline Liquiritin & $y=3.9428 x+0.0289$ & 0.9996 & $0.25 \sim 2$ \\
Isoliquiritin & $y=8.6091 x-0.0137$ & 0.9999 & $0.3 \sim 2$ \\
Liquiritigenin & $y=2.3963 x-0.0132$ & 0.9997 & $0.3 \sim 2$ \\
\hline
\end{tabular}

\section{Results and Discussion}

3.1. Selection of Solvent and Internal Standard. The results showed that the Glycyrrhiza uralensis Fisch. extract had good solubility in DMSO- $\mathrm{d}_{6}$ solvent through the preliminary experiment. Besides, the target analytes were stable and easy to separate in the solvent. Therefore, DMSO- $\mathrm{d}_{6}$ was selected as the solvent for qNMR analysis.

As a qNMR experiment, the internal standard should have high stability, high purity, easy weighing nature, and no effect on the peaks of the materials to be tested. According to the previous work experience, cresol, pyrazine, maleic acid, and dichloromethane were selected for experiments. The results showed that only dichloromethane had good stability during the experiment and was able to overcome peak-to-peak mutual influence. Therefore, dichloromethane was selected as the internal standard. 
TABLe 2: Recovery (\%) of the flavonoids in Glycyrrhiza uralensis Fisch. by the ${ }^{1} \mathrm{H}$-qNMR method.

\begin{tabular}{cccccc}
\hline & \multicolumn{2}{c}{ Liquiritin } & \multicolumn{2}{c}{ Isoliquiritin } & \multicolumn{2}{c}{ Liquiritigenin } \\
& Recovery & RSD & Recovery & RSD & Recovery (\%) \\
\hline 1 & 106.35 & & 101.09 & 107.32 & 1.86 \\
2 & 108.80 & 1.16 & 100.00 & 1.66 & 107.38 \\
3 & 108.00 & & 103.30 & & 110.83 \\
\hline
\end{tabular}

TABLE 3: Content (\%) of three flavonoids in Glycyrrhiza uralensis Fisch. by the ${ }^{1} \mathrm{H}$-qNMR method.

\begin{tabular}{lccc}
\hline Sample & Liquiritin (\%) & Liquiritigenin (\%) & Isoliquiritin (\%) \\
\hline Sample A & 0.24 & 0.11 & 0.18 \\
Sample B & 0.50 & 0.18 & 0.30 \\
Sample C & 0.17 & 0.05 & 0.01 \\
Sample D & 0.38 & 0.07 & 0.09 \\
Sample E & 1.92 & 0.22 & 0.29 \\
Sample F & 0.29 & 0.14 & 0.13 \\
\hline
\end{tabular}

Note. Sample A (Hangqi), Sample B (Yanchi), Sample C (Longxi), Sample D (Baotou), Sample E (Chifeng), and Sample F (Taklamakan Desert).

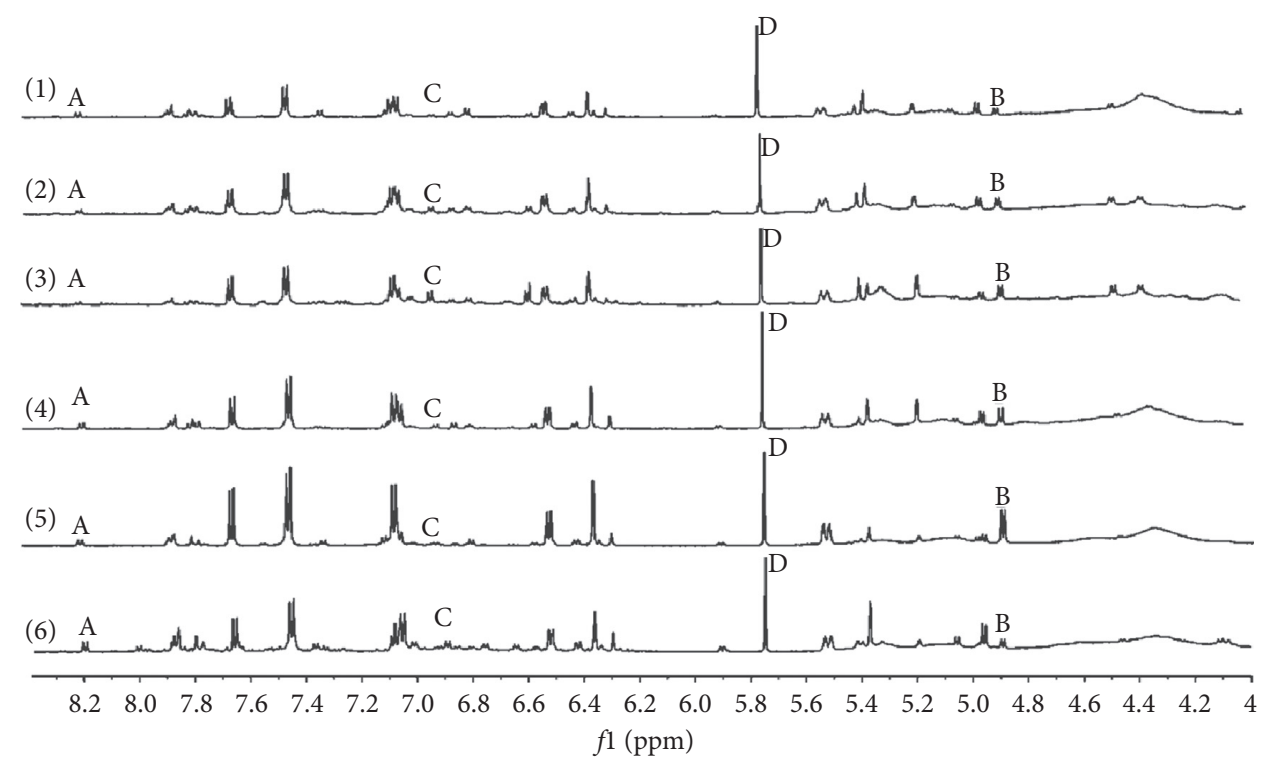

FIGURE 3: ${ }^{1} \mathrm{H}-\mathrm{NMR}$ spectra of Glycyrrhiza uralensis Fisch. from different regions: Hangqi (1); Yanchi (2); Longxi (3); Baotou (4); Chifeng (5); Taklamakan Desert (6). The signals of four contents were at $\delta 8.187-\delta 8.172 \mathrm{ppm}$ for isoliquiritin (A), $\delta 4.891-\delta 4.878 \mathrm{ppm}$ for liquiritin. (B), $\delta 6.790-\delta 6.776 \mathrm{ppm}$ for liquiritigenin (C), and $\delta 5.75 \mathrm{ppm}$ for dichloromethane (D).

3.2. Choice of Relaxation Delays. The instrument parameters such as D1 have a greater impact on the accuracy of the test results. Studies have shown that D1 must be set long enough ( $>5 \mathrm{~T} 1$ ) in qNMR experiments to fully relax the nucleus and then be integrated. The impact of D1 on the experimental results was carried out. For the same sample to be tested, using the ratio of the quantitative peak and the internal standard peak (Au/As) as a reference, the D1 values for liquiritin, liquiritigenin, and isoliquiritin were $5 \mathrm{~s}, 10 \mathrm{~s}, 20 \mathrm{~s}$, $30 \mathrm{~s}$, and $40 \mathrm{~s}$. When the D1 value is greater than $10 \mathrm{~s}$, the $\mathrm{Au} /$ As value will not change anymore. Hence, $10 \mathrm{~s}$ was select as relaxation delays to save test time.
3.3. Quantitative Results. The contents of three flavonoids in Glycyrrhiza uralensis Fisch. were determined by the ${ }^{1} \mathrm{H}$ qNMR for the first time, and the results are shown in Table 3 and Figure 3.

The experimental result showed that Glycyrrhiza uralensis Fisch. from Chifeng, Inner Mongolia, had the highest content of flavonoids.

The HPLC method was used to verify the ${ }^{1} \mathrm{H}$-qNMR. The above extract was accurately weighed to $10.00 \mathrm{mg}$ and completely dissolved in $10 \mathrm{~mL}$ of $70 \%$ absolute ethanol by ultrasonic treatment which was transferred to a sample vial for HPLC determination. Each batch of plant material 
TABLE 4: Comparison of contents of three components between ${ }^{1} \mathrm{H}$-qNMR and HPLC.

\begin{tabular}{ccccccc}
\hline & \multicolumn{2}{c}{ Liquiritin } & \multicolumn{2}{c}{ Isoliquiritin } & \multicolumn{2}{c}{ Liquiritigenin } \\
& HPLC & NMR & HPLC & NMR & HPLC (\%) & NMR (\%) \\
\hline 1 & 16.32 & 16.68 & 4.63 & 4.59 & 0.87 & 0.94 \\
2 & 16.30 & 16.65 & 4.66 & 4.60 & 0.88 & 0.93 \\
3 & 16.32 & 16.66 & 4.64 & 4.60 & 0.90 & 0.94 \\
\hline
\end{tabular}

was performed in triplicate. The ${ }^{1} \mathrm{H}$-qNMR method was also used to determine the composition of three batches of plant materials, each batch of samples was repeated 3 times, and the results were compared with HPLC. The details of the HPLC are shown in Supplementary Materials. The results are shown in Table 4. There was no significant difference between the two measurement methods.

\section{Conclusion}

In this study, a ${ }^{1} \mathrm{H}$-qNMR method was developed for the simultaneous determination of liquiritin, isoliquiritin, and liquiritigeninin in Glycyrrhiza uralensis Fisch. for the first time. This work provided a new effective method for quality control of Glycyrrhiza uralensis Fisch.

\section{Data Availability}

The original ${ }^{1} \mathrm{H}-\mathrm{NMR}$ spectral data and the analysis method of the data used to support the findings of this study are available from the corresponding authors upon request.

\section{Conflicts of Interest}

The authors declare that they have no conflicts of interest.

\section{Authors' Contributions}

Ping Yu and Qian Li have contributed equally to this work.

\section{Acknowledgments}

This work was supported by the Research Program Sponsored by Discipline Construction Fund Project of Gansu Agricultural University (GSAU-XKJS-2018-086), the National Natural Science Foundation of China (31860102), and Gansu Provincial Key Laboratory of Aridland Crop Science (No. GSCS-2018-3).

\section{Supplementary Materials}

Supplementary Materials are about "Sample Analysis and Verification by HPLC". Figure 1: HPLC chromatograms of medicinal material extract (A) and mixed reference solution (B). Table 1: regression data for three bioactive compounds obtained by HPLC results. Table 2: the precision, repeatability, and stability of three flavonoids. Table 3 : the recovery of three flavonoids. Table 4: comparison of H-qNMR and HPLC. (Supplementary Materials)

\section{References}

[1] Q. Zhang and M. Ye, "Chemical analysis of the Chinese herbal medicine Gan-Cao (licorice)," Journal of Chromatography A, vol. 1216, no. 11, pp. 1954-1969, 2009.

[2] S. Zhou, J. Cao, F. Qiu, W. Kong, S. Yang, and M. Yang, "Simultaneous determination of five bioactive components in radix glycyrrhizae by pressurised liquid extraction combined with UPLC-PDA and UPLC/ESI-QTOF-MS confirmation," Phytochemical Analysis, vol. 24, no. 6, pp. 527-533, 2013.

[3] Y. H. Kim, E. K. Shin, D. H. Kim, H. H. Lee, J. H. Y. Park, and J.-K. Kim, "Antiangiogenic effect of licochalcone A," Biochemical Pharmacology, vol. 80, no. 80, pp. 1152-1159, 2010.

[4] Y. Sun and A. Wumaier, "Research progress on extraction methods and pharmacological effects of licorice flavonoids," Xinjiang Journal of Traditional Chinese Medicine, vol. 27, pp. 72-75, 2009.

[5] X. Y. Gao, W. Q. Wang, and S. L. Wei, "Review of pharmacological effects of glycyrrhiza radix and its bioactive compounds," Zhong Guo Zhong Yao Za Zhi, vol. 34, no. 6, pp. 2695-2700, 2009.

[6] M. N. Asl and H. Hosseinzadeh, "Review of pharmacological effects ofGlycyrrhiza sp. and its bioactive compounds," Phytotherapy Research, vol. 22, no. 6, pp. 709-724, 2008.

[7] K. H. Jung, J. Y. Seo, and H.-J.. Suh, "Antioxidant activities of licorice-derived prenylflavonoids," Nutrition Research and Practice, vol. 6, pp. 491-498, 2012.

[8] X. Li and W. Li, "Research progress on the pharmacological effects of the active ingredient of licorice extract," Jiangsu Journal of Traditional Chinese Medicine, vol. 51, no. 5, pp. 81-86, 2019.

[9] B. H. Chen and Y. Gao, "Application of licorice and its products in toothpaste," Oral Care Industry, vol. 27, no. 6, pp. 6-8, 2017.

[10] Y.-C. Xie, X.-W. Dong, X.-M. Wu, X.-F. Yan, and Q.-M. Xie, "Inhibitory effects of flavonoids extracted from licorice on lipopolysaccharide-induced acute pulmonary inflammation in mice," International Immunopharmacology, vol. 9, no. 2, pp. 194-200, 2009.

[11] J. Vaya, P. A. Belinky, and M. Aviram, "Antioxidant constituents from licorice roots: isolation, structure elucidation and antioxidative capacity toward LDL oxidation," Free Radical Biology and Medicine, vol. 23, no. 2, pp. 302-313, 1997.

[12] R. Roulard, J.-X. Fontaine, A. Jamali et al., "Use of qNMR for speciation of flaxseeds (linum usitatissimum) and quantification of cyanogenic glycosides," Analytical and Bioanalytical Chemistry, vol. 409, no. 30, pp. 7011-7026, 2017.

[13] O. K. Adebayo and D. Donaldson, "Liquorice and its health implications," Journal of the Royal Society for the Promotion of Health, vol. 120, no. 2, pp. 83-89, 2000.

[14] X. J. Chen, J. Zhao, Q. Meng, S. P. Li, and Y. T. Wang, "Simultaneous determination of five flavonoids in licorice using pressurized liquid extraction and capillary electrochromatography coupled with peak suppression diode array detection," Journal of Chromatography A, vol. 1216, no. 43, pp. 7329-7335, 2009.

[15] N. C. Zhou, Y. Zou, and M. L. Qin, “A simple method for evaluation pharmacokinetics of glycyrrhetinic acid and potential drug-drug interaction between herbal ingredients," Scientific Reports, vol. 9, no. 1, p. 11308, 2019.

[16] P. Liu, H. Yang, F. Long et al., "Bioactive equivalence of combinatorial components identified in screening of an 
herbal medicine," Pharmaceutical Research, vol. 31, no. 7, pp. 1788-1800, 2014.

[17] S. K. Bharti and R. Roy, "Quantitative ${ }^{1}$ H NMR spectroscopy," TrAC Trends in Analytical Chemistry, vol. 35, pp. 5-26, 2012.

[18] F. Malz and H. Jancke, "Validation of quantitative NMR," Journal of Pharmaceutical and Biomedical Analysis, vol. 38, no. 5, pp. 813-823, 2005.

[19] H. Gadape and K. Parikh, "Quantitative determination and validation of carvedilol in pharmaceuticals using quantitative nuclear magnetic resonance spectroscopy," Analytical Methods, vol. 3, no. 10, pp. 2341-2347, 2011.

[20] T. S. Al-Deen, D. B. Hibbert, J. M. Hook, and R. J. Wells, “An uncertainty budget for the determination of the purity of glyphosate by quantitative nuclear magnetic resonance (QNMR) spectroscopy," Accreditation and Quality Assurance, vol. 9, no. 1-2, pp. 55-63, 2004.

[21] Q. Liang, Q. Wang, Y. Wang, Y.-n. Wang, J. Hao, and M. Jiang, "Quantitative ${ }^{1} \mathrm{H}-\mathrm{NMR}$ spectroscopy for profiling primary metabolites in mulberry leaves," Molecules, vol. 23, no. 3, p. 554, 2018.

[22] D. S. Araújo, A. P. Oliveira, and C. A. Santos, "Total content of kaurene diterpenes in Annona vepretorum stems via ${ }^{1} \mathrm{H}$-qNMR: a method for speeding the identification of bioactive extracts," Phytochemical Analysis, vol. 30, no. 1, pp. 83-88, 2019.

[23] G. K. Webster and S. Kumar, "Expanding the analytical toolbox: pharmaceutical application of Quantitative NMR," Analytical Chemistry, vol. 86, no. 23, pp. 11474-11480, 2014.

[24] R. J. Wells, J. M. Hook, T. S. Al-Deen, and D. B. Hibbert, "Quantitative nuclear magnetic resonance (QNMR) spectroscopy for assessing the purity of technical grade agrochemicals: 2,4-dichlorophenoxyacetic acid (2,4-D) and sodium 2,2-dichloropropionate (dalapon sodium)," Journal of Agricultural and Food Chemistry, vol. 50, no. 12, pp. 33663374, 2002.

[25] G. F. Pauli, "qNMR ? A versatile concept for the validation of natural product reference compounds," Phytochemical Analysis, vol. 12, no. 1, pp. 28-42, 2001.

[26] S. Lin, J. Su, and Q. Ye, "Application progress in quantitative NMR in analysis of traditional Chinese medicine," Journal of Pharmaceutical Practice, vol. 32, no. 2, pp. 92-95, 2014.

[27] A. Owczarek, A. Kłys, and M. A. Olszewska, "A validated ${ }^{1} \mathrm{H}$ qNMR method for direct and simultaneous quantification of esculin, fraxin and (-)-epicatechin in Hippocastani cortex," Talanta, vol. 192, pp. 263-269, 2019.

[28] B. Avula, V. C. Joshi, Y.-H. Wang, and I. A. Khan, "Simultaneous identification and quantification of anthraquinones, polydatin, and resveratrol in polygonum multiflorum, various polygonum species, and dietary supplements by liquid chromatography and microscopic study of polygonum species," Journal of AOAC International, vol. 90, no. 6, pp. 1532-1538, 2007.

[29] R. A. F. Cavalcante, F. L. Silva, F. Favero, I. S. Resck, A. L. Pereira, and A. H. L. Machado, "Quantitative $1 \mathrm{H} \mathrm{NMR}$ spectroscopy (qNMR) in the early process development of a new quorum sensing inhibitor," Magnetic Resonance in Chemistry, vol. 58, no. 1, pp. 31-40, 2020.

[30] N. Duangdee, N. Chamboonchu, S. Kongkiatpaiboon, and S. Prateeptongkum, "Quantitative ${ }^{1}$ HNMR spectroscopy for the determination of oxyresveratrol in Artocarpus lacucha heartwood," Phytochemical Analysis, vol. 30, no. 6, pp. 617-622, 2019.

[31] D. Bertelli, V. Brighenti, L. Marchetti, A. Reik, and F. Pellati, "Nuclear magnetic resonance and high-performance liquid chromatography techniques for the characterization of bioactive compounds from Humulus lupulus L. (hop)," Analytical and Bioanalytical Chemistry, vol. 410, no. 15, pp. 3521-3531, 2018.

[32] R. Cao, A. Nonaka, F. Komura, and T. Matsui, "Application of diffusion ordered-1H-nuclear magnetic resonance spectroscopy to quantify sucrose in beverages," Food Chemistry, vol. 171, no. 171, pp. 8-12, 2015.

[33] T. Saito, T. Ihara, M. Koike et al., "A new traceability scheme for the development of international system-traceable persistent organic pollutant reference materials by quantitative nuclear magnetic resonance," Accreditation and Quality Assurance, vol. 14, no. 2, pp. 79-86, 2009.

[34] L. Yang, Q. Li, Y. M. Feng, and D. Y. Qiu, "Simultaneous determination of three coumarins in Angelica dahurica by ${ }^{1} \mathrm{H}$-qNMR method: a fast and validated method for crude drug quality control," Journal of Analytical Methods in Chemistry, vol. 2020, Article ID 8987560, 7 pages, 2020.

[35] Y. M. Feng, L. Yang, Q. Li, and D. Y. Qiu, "The use of ${ }^{1} \mathrm{H}-$ qNMR method for simultaneous determination of osthol, columbianadin, and isoimperatorin in Angelicae Pubescentis radix," Journal of AOAC International, vol. 103, no. 2, pp. 851-856, 2020.

[36] Chinese Pharmacopoeia Commission, Pharmacopoeia of People's Republic of China, China Medical Science and Technology Press, Beijing, China, 2015.

[37] M. A. Farag, A. Porzel, and L. A. Wessjohann, "Comparative metabolite profiling and fingerprinting of medicinal licorice roots using a multiplex approach of GC-MS, LC-MS and 1D NMR techniques," Phytochemistry, vol. 76, pp. 60-72, 2012.

[38] C. Simmler, D. Nikolić, D. C. Lankin et al., "Orthogonal analysis underscores the relevance of primary and secondary metabolites in licorice," Journal of Natural Products, vol. 77, no. 8, pp. 1806-1816, 2014. 